\title{
DIAGNÓSTICO SITUACIONAL DEL CÁNCER DE MAMA EN LA CIUDAD DE CÚCUTA Y AREA METROPOLITANA EN EL PERÍODO 2002 - 2003
}

Por:

Angélica Maria Restrepo Jurado' *, Johanna Carolina Guerrero Franco', Joysi Marilyn Sánchez Romero', Leonardo Contreras Parada²

\section{RESUMEN}

Para determinar la situación actual del cáncer de mama en la ciudad de Cúcuta y su área metropolitana se realizó un estudio descriptivo con 201 pacientes que fueron diagnosticadas en las distintas instituciones de la ciudad durante el periodo 2002 - 2003. En primer lugar se analizó la historia clínica, donde se definieron como variables del estudio: incidencia, sociodemográficas, factores de riesgo, diagnósticos, estadificación y tratamiento de la enfermedad. La incidencia para el año 2003 disminuyo en un 10\% en relación al año anterior, la población de escasos recursos es la más afectada, la edad de aparición por encima de los 40 años abarco el $85 \%$ de los casos. Tanto los factores de riesgo como los protectores no fueron significativos ante la aparición de la enfermedad. Gran proporción de la población fue diagnosticada en estadios avanzados con tamaño promedio de los tumores de $4.9 \mathrm{~cm}$., siendo el resultado de diagnósticos tardíos. El tratamiento más utilizado es la mastectomía radical modificada, quimioterapia prequirúrgica, radioterapia Postquirúrgica.

En segundo lugar se analizaron los conocimientos sobre detección precoz del cáncer de mama a las pacientes diagnosticadas con la enfermedad y a sus hijas, las cuales presentaron un mejor nivel de conocimientos debido al enfrentamiento de la enfermedad por su familia, realizándose posteriormente un programa educativo "Hablemos de cáncer de mama" con enfoque familiar.
Palabras claves: Diagnóstico Situacional, Cáncer de mama, Autoexamen de mama, Mamografía

\section{INTRODUCCIÓN}

El cáncer de mama con sus orígenes inciertos y consecuencias devastadoras ha sido un gran punto de atención. El hecho de que la glándula mamaria sea un órgano par (doble) aumenta la exposición a la enfermedad, BLAND (1993). En Colombia ocupa la tercera causa de muerte por tumores malignos; es una enfermedad que presenta alta incidencia acompañada de diagnósticos en estadios avanzados, provocando tratamientos largos, costosos, o por el contrario conducir a la fatalidad, clasificándose como determinante problema de salud pública.

En el cáncer de mama se hace necesario replantear la perspectiva actual del manejo de la entidad iniciando por el reconocer que la comunidad es pobre en educación preventiva ya que los programas hoy planteados no ejercen el impacto necesario para crear conciencia y/o en la mayoría de los casos la población vulnerable no cuenta con acceso a la información, prolongando el tiempo transcurrido entre el inicio del cuadro y la atención de éste; complicando el pronóstico de la enfermedad.

La profesión de enfermería debe comprometerse aun más en la canalización de la población diseñando y aplicando estrategias educacionales que lleguen a la población en general.

El presente estudio busca dar a conocer cual es la situación en la que se encuentra la ciudad de Cúcuta y su Área Metropolitana frente al cáncer de mama,

\footnotetext{
'Grupo de Investigación en Salud Pública, Universidad Francisco de Paula Santander. * angelicresju@yahoo.es

${ }^{2}$ Médico Cirujano-Asociacón Nortesantanderana de Cirugía
} 
Diagnóstico situacional del cáncer de mama en la ciudad de Cúcuta y Área Metropolitana en el período 2002 - 2003

Angélica María Restrepo Jurado, Johanna Carolina Guerrero Franco, Joysi Marilyn Sánchez Romero

indagando la influencia de los factores de riesgo, el estadio en que se encuentran, estudios complementarios, tratamiento brindado, así como los conocimientos de las mujeres diagnosticadas y mujeres con factor de riesgo frente a la detección temprana del cáncer de mama, en busca de plantear y dar solución a los mismos.

\section{MATERIALES Y METODOS}

El grupo investigador revisó el total de historias clínicas de las pacientes diagnosticadas con cáncer de mama durante el periódo 2002 -2003 en instituciones públicas y privadas que permitieran la recopilación de la información, definiendo variables sociodemográficas, factores de riesgo, antecedentes personales y familiares, localización tumoral, clasificación del cáncer, medios diagnósticos y tratamiento.

Obtenida la información de la historia clínica se procedía a la identificación del domicilio por vía telefónica acordando con la paciente la fecha de la visita, si accedía a que se realizara; en casos en donde el dato telefónico fue incorrecto, se encontrara fuera de servicio o simplemente no contará con él, se procedía a realizar directamente la visita domiciliaria, informando cual era el motivo de la visita.

Inicialmente se daba a conocer el consentimiento informado, una vez autorizara se procedía a la segunda parte del estudio aplicando dos instrumentos, el primero para las mujeres con diagnóstico de cáncer de mama, indagando sobre los conocimientos y/o actitudes antes de presentar la enfermedad respecto a técnicas de detección precoz. El segundo instrumento aplicado a las mujeres con factor de riesgo, de igual manera media los conocimientos y/o actitudes que presentan sobre la detección precoz del cáncer de mama. Tomando un máximo de dos hijas naturales de las mujeres diagnosticadas que se encontrarán en el domicilio.
Una vez aplicados los instrumentos se llevó a cabo la ejecución del programa educativo "Hablemos de cáncer de mama " para lo cual se diseñó un folleto educativo didáctico que reforzará la educación brindada por el grupo investigador y que en un momento dado permitiera ser consulta en caso de dudas, tratándose aspectos relacionados con las características normales de la mama, definición del cáncer de mama, factores de riesgo, señales de alarma, técnicas de detección precoz, profundizando en el autoexamen de mama como la técnica más económica y que se encuentra en todo momento al alcance de la mujer.

La información obtenida fue sometida a un análisis descriptivo, en donde se puede obtener una aproximación de la incidencia del cáncer de mama en la ciudad de Cúcuta y área metropolitana, variables sociodemográficas de la población diagnosticada, el manejo que se esta brindando, así como los conocimientos de la población referente a la entidad. Esperando que la información aportada conlleve al fortalecimiento de los programas de promoción y prevención del cáncer de mama.

\section{RESULTADOS}

- Se creó una ficha para la recolección de información sobre los casos de cáncer de mama en la ciudad de Cúcuta y su Área Metropolitana en el período comprendido entre enero de 2002 a diciembre de 2003; el número de casos encontrados y que se dispusieron a estudio fue de 201 , de los cuales $55.22 \%$ se presentaron en el año 2002 y $44.78 \%$ en el siguiente año; observando una tasa de incidencia del año 2002 (2.4 X 10.000 Habitantes), y del año 2003 (1.9 X 10.000 Habitantes). Los casos de cáncer de mama presentados en el estudio disminuyeron en $10 \%$ del año 2002 al año 2003. La edad de aparición de la enfermedad corresponde a 54.9 años, donde más del $87 \%$ de las pacientes son mayores de 40 años), donde el riesgo de desarrollar la enfermedad aumenta después de la cuarta década de vida HERNANDEZ(1993). 
Diagnóstico situacional del cáncer de mama en la ciudad de Cúcuta y Área Metropolitana en el período 2002 - 2003

Angélica María Restrepo Jurado, Johanna Carolina Guerrero Franco, Joysi Marilyn Sánchez Romero

- En cuanto al tamaño del tumor, se logró recopilar información de 118 casos; determinándose que el tamaño promedio del tumor es 4.9 centímetros, con una desviación estándar 4,27 centímetros. Al compararlo con el estudio en la clínica San Pedro Claver del ISS de Bogotá, el 44\% de los casos el tamaño tumoral es superior a 5 centímetros, lo que demuestra que nuestro departamento se están diagnosticando tumores de menor tamaño $(2-5 \mathrm{~cm})$ lo que favorece que no se presente un mayor número de complicaciones.

Tabla 1. Distribucion de casos según estudios de extensión y metástasis

\begin{tabular}{|l|c|c|c|c|c|c|}
\hline \multirow{2}{*}{$\begin{array}{c}\text { ESTUDIOS DE } \\
\text { EXTENSION }\end{array}$} & \multicolumn{4}{|c|}{ METASTASIS } & \multicolumn{3}{c|}{ TOTAL } \\
\cline { 2 - 8 } & SI & $\%$ & NO & $\%$ & $\begin{array}{c}\text { No } \\
\text { CASOS }\end{array}$ & $\%$ \\
\hline Gamagrafía & 21 & 18.92 & 90 & 81.08 & 111 & 55.22 \\
\hline Radiografía de Tórax & 11 & 10.58 & 93 & 89.42 & 104 & 51.74 \\
\hline Ecografía Hepática & 9 & 8.57 & 96 & 91.43 & 105 & 52.24 \\
\hline
\end{tabular}

Gráfico 1. Distribución de casos según estudios de extensión y metástasis

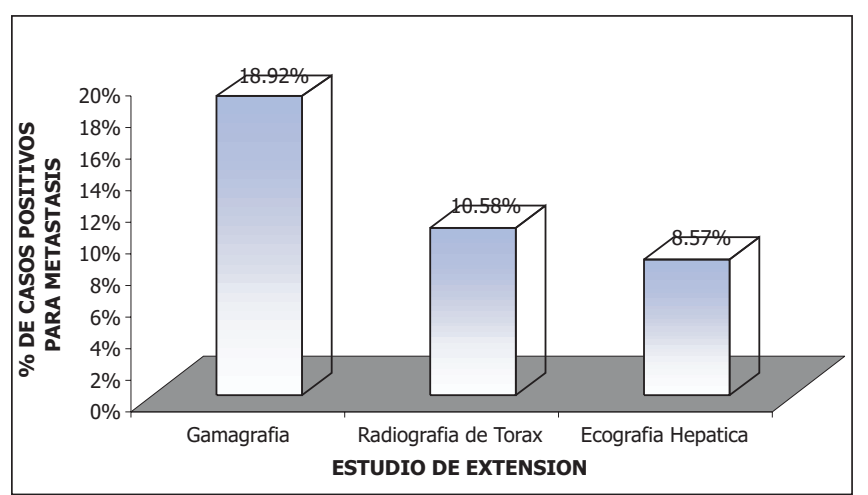

- Se encontró que de los estudios de extensión cerca del $45 \%$ de los casos no se les realizó ningún examen en búsqueda de metástasis a distancia, lo cual influye en la verdadera estratificación y por consiguiente en el pronóstico y tratamiento de la enfermedad. A pesar de esto el método más utilizado es la Gamagrafía ósea, debido a que es uno de los principales lugares de metástasis GONZÁLEZ (2000); se realizó al $55.22 \%$ de los casos, de los cuales el $18.92 \%$ presentaron resultado positivo para metástasis ósea; se encontró registro de radiografía de tórax en el $51.74 \%$ de los casos, de los cuales el $10.58 \%$ dieron positivo para metástasis. Por último, se pudo conocer que al 52.24 $\%$ de las pacientes se les realizó ecografía hepática, encontrándose que de estas, el 8.57 \% presentaron metástasis hepática.

Tabla 2. Distribucion de casos según tratamiento y estadio de la enfermedad

\begin{tabular}{|l|c|c|c|c|c|c|c|c|}
\hline \multirow{3}{*}{ ESTADIO } & \multicolumn{2}{|l|}{ TRATAMIENTO } \\
\cline { 2 - 10 } & $\begin{array}{l}\text { Radioterapia } \\
\text { Prequirúrgica }\end{array}$ & \multicolumn{2}{|l|}{$\begin{array}{c}\text { Radioterapia } \\
\text { Postquirúrgica }\end{array}$} & \multicolumn{2}{|c|}{$\begin{array}{l}\text { Quimioterapia } \\
\text { Prequirúrgica }\end{array}$} & \multicolumn{2}{|c|}{$\begin{array}{c}\text { Quimioterapia } \\
\text { Postquirúrgica }\end{array}$} \\
\cline { 2 - 10 } & No & $\%$ & No & $\%$ & No & $\%$ & No & $\%$ \\
\hline I & 1 & 3.8 & 4 & 5.5 & 2 & 1.9 & 2 & 2.7 \\
\hline II A & 3 & 11.5 & 15 & 20.5 & 23 & 21.9 & 18 & 24.0 \\
\hline II B & 1 & 3.8 & 13 & 17.8 & 14 & 13.3 & 12 & 16.0 \\
\hline III A & & & 6 & 8.2 & 9 & 8.6 & 8 & 10.7 \\
\hline III B & 8 & 30.8 & 21 & 28.8 & 32 & 30.5 & 27 & 36.0 \\
\hline IV & 5 & 19.2 & 9 & 12.3 & 13 & 12.4 & 4 & 5.3 \\
\hline SIN DATO & 8 & 30.8 & 5 & 6.8 & 12 & 11.4 & 4 & 5.3 \\
\hline
\end{tabular}

En relación a los estadios de la enfermedad, el estadio III B es el de mayor incidencia (26.87\%), seguido por el II A con $18.91 \%$; en concordancia con el estudio realizado en la clínica San Pedro Claver del ISS de Bogotá que evaluaba las características personales, parámetros pronósticos, tratamiento y sobreviva de los pacientes, donde se encontró que el estadio de mayor frecuencia es el IIIB. Al analizar la información de los casos de acuerdo al estadio del cáncer y al tratamiento realizado, observamos que la mayoría de las quimioterapias prequirúrgicas se realizaron a pacientes con estadio III B. Situación similar ocurre con las radioterapias posquirúrgicas y las quimioterapias, donde la mayoría de estas, se realizaron a pacientes con estadio de la enfermedad superior o igual a III B. Proporcionalmente se puede decir que el tratamiento de mayor incidencia que se realizó a las pacientes en un estadio avanzado de la enfermedad fue la quimioterapia prequirúrgica por la necesidad de reducir el tamaño tumoral para la posterior cirugía. También es de resaltar que la radioterapia no solo 
Diagnóstico situacional del cáncer de mama en la ciudad de Cúcuta y Área Metropolitana en el período 2002 - 2003

Angélica María Restrepo Jurado, Johanna Carolina Guerrero Franco, Joysi Marilyn Sánchez Romero

reduce la tasa de recidiva local sino que incrementa el tiempo libre de recidiva así como la supervivencia, HAAGENSEN(1971). En el estudio realizado por el Instituto Canario de Investigación del Cáncer se demostró que las pacientes que reciben quimioterapia más radioterapia o hormonoterapia más radioterapia tiene menores tasas de recidiva loco regional y mayor supervivencia global que las pacientes que solo reciben quimioterapia $u$ hormonoterapia.

Al revisar la información recopilada en cuanto al régimen de afiliación al sistema general de seguridad social en salud y el estadio de la enfermedad, la mayoría de los casos de pacientes vinculadas y/o subsidiadas se les diagnosticó la enfermedad en un estadio avanzado o superior a III B; caso contrario ocurre con los casos de pacientes que pertenecen al régimen contributivo, excepciones y/o particular tienen diagnóstico de la enfermedad con un estadio temprano o inferior a III.

Tabla 3. Distribución de casos según mamografía

\begin{tabular}{|l|c|c|}
\hline \multicolumn{1}{|r|}{ MAMOGRAFIA } & No DE CASOS & $\%$ \\
\hline SI & 80 & 39.80 \\
\hline NO & 77 & 38.31 \\
\hline SIN DATO & 44 & 21.89 \\
\hline TOTAL & 201 & 100 \\
\hline & & \\
\hline
\end{tabular}

Gráfico 2. Distribucion de casos según mamografía

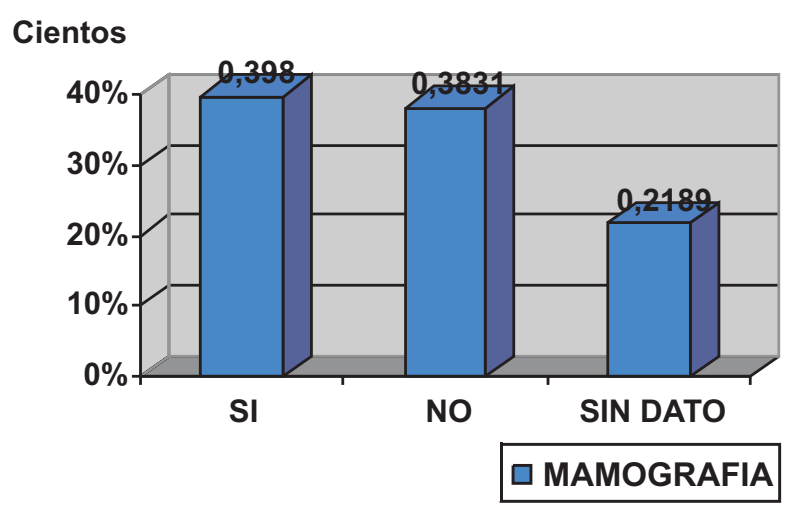

Al analizar la mamografía como método diagnóstico se determinó que el $39.8 \%$ de los casos se les realizó dicho estudio, mientras que al $38.31 \%$ no se les practicó este examen, encontrándose que estas proporciones son muy similares a pesar de su importancia como método de detección precoz, diagnóstico y seguimiento posterior a la quimioterapia, BLAND(1993).

\section{CONOCIMIENTOS Y ACTITUDES DE LAS MUJERES CON DIAGNOSTICO Y MUJERES CON FACTOR DE RIESGO}

- Se realizó una encuesta con el fin de determinar los conocimientos y/o actitudes sobre detección precoz del cáncer de mama que tenían las mujeres con este diagnóstico antes de presentarla, así como los conocimientos y/o actitudes que presentan las mujeres con factor de riesgo que son hijas de la ya diagnosticadas. Por motivos de accesibilidad y disponibilidad solo se pudo realizar la encuesta 25 mujeres para las de diagnóstico de las cuales el $52 \%$ casadas, el $24 \%$ son viudas y otro $20 \%$ están solteras. El $4 \%$ restante son mujeres separadas y 22 mujeres para las de factor de riesgo de las cuales la mayoría son solteras con $72.7 \%$ y $27.3 \%$ de ellas están casadas o se encuentran en unión libre.

Tabla 4. Clasificacion de los conocimientos y las actitudes de las mujeres con diagnóstico y mujeres con factor de riesgo de cáncer de mama

\begin{tabular}{|c|c|c|c|c|}
\hline \multirow{3}{*}{$\begin{array}{l}\text { COMPORTAMIENTOS } \\
\text { Y/O ACTITUDES }\end{array}$} & \multicolumn{4}{|c|}{ GRUPO ESTUDIO } \\
\hline & \multicolumn{2}{|c|}{$\begin{array}{c}\text { MUJERES CON } \\
\text { DIAGNOSTICO DE } \\
\text { CANCER DE MAMA }\end{array}$} & \multicolumn{2}{|c|}{$\begin{array}{c}\text { MUJERES CON } \\
\text { FACTOR DE RIESGO } \\
\text { DE CANCER DE MAMA }\end{array}$} \\
\hline & No & $\%$ & No & $\%$ \\
\hline INADECUADAS & 13 & 52 & 3 & 13,6 \\
\hline $\begin{array}{l}\text { MEDIANAMENTE } \\
\text { ADECUADAS }\end{array}$ & 9 & 36 & 11 & 50 \\
\hline ADECUADAS & 3 & 12 & 8 & 36,4 \\
\hline TOTAL & 25 & 100 & 22 & 100 \\
\hline
\end{tabular}

Revista Respuestas - Universidad Francisco de Paula Santander Año 9 No. 2 
Diagnóstico situacional del cáncer de mama en la ciudad de Cúcuta y Área Metropolitana en el período 2002 - 2003 Angélica María Restrepo Jurado, Johanna Carolina Guerrero Franco, Joysi Marilyn Sánchez Romero

Gráfico 3. Clasificación de los conocimientos y actitudes de las mujeres con diagnóstico y mujeres con factor de riesjo de cáncer de mama

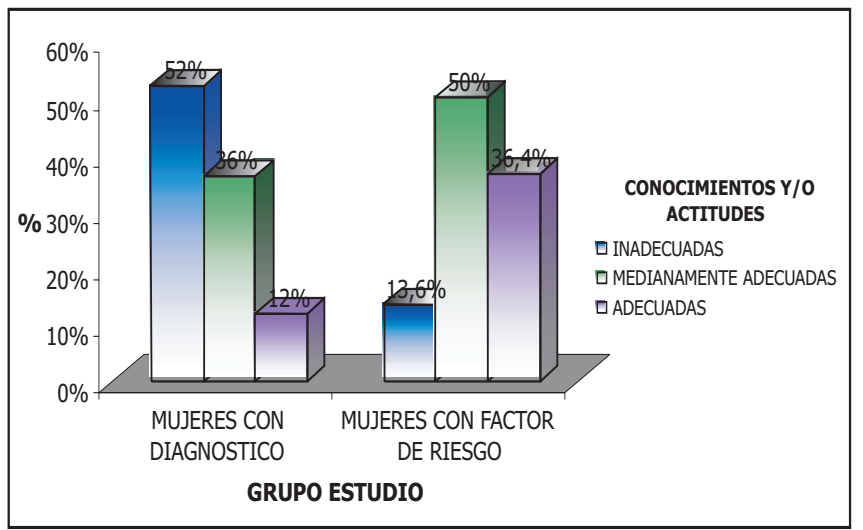

- Se puede observar que las mujeres con el factor de riesgo de cáncer de mama por antecedente familiar de primer grado de consanguinidad, tienen mejores conocimientos, que las pacientes con diagnóstico antes de presentar la enfermedad, demostrando que la experiencia influye en la sensibilización de la detección temprana de la entidad, pero aunque se obtengan mejores conocimientos en las mujeres con factor de riesgo ninguna expresa con total claridad estas medidas.

La ausencia de implementación de programas educativos en prevención del cáncer de mama generan confusiones en el manejo de información por parte de la población, donde la técnica del autoexamen de mama no se esta realizando de la manera establecida y bajo las recomendaciones indicadas.

\section{DISCUSIÓN}

Actualmente en la ciudad de Cúcuta y su Área Metropolitana se presenta una alta incidencia de pacientes con cáncer de mama en estadios relativamente avanzados, iguales o superiores a IIIB que generan una sobreviva a cinco años de solo el $44 \%$. Lo anterior es el reflejo de tamaños tumorales de consideración, compromiso de ganglios, y en algunos casos metástasis, como consecuencia de un diagnóstico tardío y estudios de extensión incompleto en todos los regímenes, por lo que complica la clasificación real y el pronostico; influyendo en la elección del tratamiento indicado y por consiguiente afectando el tiempo de sobreviva del paciente. Los índices de mayor evolución de los estadios se encuentran en los regímenes vinculados y subsidiados, lo que hace pensar que las medidas de detección temprana del cáncer de mama no están siendo adoptadas por la población debido a que existe una falta de divulgación de información que sensibilice la población sobre la enfermedad. Gran proporción de estas pacientes pertenecen al régimen subsidiado y otras son vinculadas al sistema, predominando la población con escasos recursos que presentan problemas de accesibilidad económica a los servicios de salud. En lo relacionado a los conocimientos de las técnicas de detección precoz de la población, existe una baja concientización en el reconocimiento de las señales de alarma y las recomendaciones para detectar a tiempo la enfermedad; dentro de las pacientes que realizan el autoexamen de mama solo una parte de ellas asigna la importancia necesaria y la técnica correcta en su realización.

\section{CONCLUSIONES}

- La incidencia del cáncer de mama disminuyó un 10\% para el año 2003 en relación al año anterior.

- Se establece que una gran proporción de pacientes presentan estadios avanzados, siendo reflejo de tamaños tumorales de consideración, compromiso de ganglios, y en algunos casos metástasis, como consecuencia de un diagnóstico tardío y estudios de extensión incompleto en todos los regímenes, por lo que complica la clasificación real y el pronostico; influyendo en la elección del tratamiento indicado y por consiguiente afectando el tiempo de sobreviva del paciente.

- Es de destacar que la gran utilización de la quimioterapia prequirúrgica se debe al hallazgo de tamaños tumorales significativamente grandes 
Diagnóstico situacional del cáncer de mama en la ciudad de Cúcuta y Área Metropolitana en el período 2002 - 2003

Angélica María Restrepo Jurado, Johanna Carolina Guerrero Franco, Joysi Marilyn Sánchez Romero

que hacen necesario buscar su reducción para llevar a cabo la posterior cirugía, que en su mayoría la mastectomía radical modificada es la elección, dirigida a la prevención de recurrencias locales y regionales en la pared del tórax y la axila, en donde el tratamiento se vio integrado en su mayoría con la quimioterapia y radioterapia post quirúrgica.

- Existe una baja concientización de la población en el reconocimiento de las señales de alarma y recomendaciones frente al cáncer de mama que se pueden detectar a tiempo con un diagnóstico temprano que favorecerá el pronostico de la enfermedad.

- Se presento un mejor nivel de conocimientos y/o actitudes dentro de las mujeres factor de riesgo de adquirir la enfermedad respecto a las mujeres que presentaron el diagnóstico de cáncer de mama, lo anterior es un reflejo de la experiencia generada en la familia en lo relacionado con la enfermedad generando concientización en la prevención de la enfermedad.

\section{BIBLIOGRAFIA}

BALLESTEROS, Mateus. Patología tumoral de la mama. España: Jims, 1793. 21-57 p.

BLAND Kirby y COPELAND Eduard. La mama. Tomo I. Panamericana, 1993.

GONZALEZ AGUDELO, Marco Antonio. Fundamentos de Medicina. $9^{a}$ Edición. Colombia: Corporación de Investigaciones Biológicas, 2000.

HAAGENSEN, C. Enfermedades del seno. London: Saunder Company, 1971. 223 p.

HERNANDEZ MUÑOZ, Gerardo. Avances en Mastología. Santiago de Chile: Universitaria, 1993. 